Supporting Information

\title{
Photoreaction of a 2,11-Diaza[3.3]paracyclophane Derivative: Formation of Octahedrane by Photochemical Dimerization of Benzene
}

Division of Chemistry and Biochemistry, The Graduate School of Natural Science and Technology, Okayama University, Tsushima-Naka 3-1-1, Okayama 700-8530, Japan

Hideki Okamoto, ${ }^{*}$ Kyosuke Satake, Hiroyuki Ishida and Masaru Kimura

\section{List of Contents}

S2 General aspects.

S3 Experimental details for the photolysis of cyclophane 1.

S4 CHART S1. Assignments of ${ }^{1} \mathrm{H}$ and ${ }^{13} \mathrm{C}$ NMR spectra of the photoproducts 3 and 4 .

S5 FIGURE S1. ${ }^{1} \mathrm{H}$ NMR spectrum of octahedrane 3.

S6 FIGURE S2. ${ }^{13} \mathrm{C}$ NMR spectrum of octahedrane 3.

S7 FIGURE S3. NOESY spectrum of octahedrane 3.

S8 FIGURE S4. HSQC spectrum of octahedrane 3.

S9 FIGURE S5. HMBC spectrum of octahedrane 3.

S10 FIGURE S6. ${ }^{1}$ H NMR spectrum of cage compound 4.

S11 FIGURE S7. ${ }^{13} \mathrm{C}$ NMR spectrum of cage compound 4.

S12 FIGURE S8. NOESY spectrum of cage compound 4.

S13 FIGURE S9. HSQC spectrum of cage compound 4.

S14 FIGURE S10. HMBC spectrum of cage compound 4.

S15 Summary of the X-ray crystallographic study of octahedrane 3.

S17 FIGURE S12. LUMO figures of cyclophanes 1 and $\mathbf{2}$ calculated by DFT method.

S18 FIGURE S13. Absorption and fluorescence spectra of cyclophanes 1 and 2.

S19 Additional References. 


\section{General aspects.}

The ${ }^{1} \mathrm{H}$ and ${ }^{13} \mathrm{C}$ NMR spectra were recorded using a Varian UNITY INOVA AS600 spectrophotometer at 600 and $150 \mathrm{MHz}$, respectively. The photoreactions of cyclophanes 1 and 2 were carried out using a Rayonet photoreactor equipped with RPR-300 fluorescent lamps (300 nm, $16 \times 25 \mathrm{~W})$ at room temperature. Spectroscopic grade solvents were used for the photoreactions. Absorption spectra were recorded using JASCO V530 spectrophotometer. Fluorescence spectra were measured with HITACHI F-2500 spectrophotometer in nitrogen-purged $\mathrm{MeCN}$ at $25{ }^{\circ} \mathrm{C}$ and fluorescence quantum yield was determined by using $p$-xylene in EtOH $\left(\Phi_{\mathrm{F}}=0.24\right)$ as a reference. ${ }^{1}$ The AM1 and DFT calculations were performed by using Gaussian03 package ${ }^{2}$ and the molecular orbitals were visualized with Chem3D (Cambridgesoft). 
Photolysis of cyclophane 1. Four solutions of cyclophane 1 ( 4 × $21.5 \mathrm{mg}, 4$ x $50 \mu \mathrm{mol})$ in benzene $(4 \times 10 \mathrm{ml})$ were purged with nitrogen and irradiated at $300 \mathrm{~nm}$ and room temperature in quartz glass tubes for $36 \mathrm{~h}$. The solutions were combined and concentrated under reduced pressure. The residue was separated repeatedly by preparative TLC (silica gel, $\left.\mathrm{CHCl}_{3}\right)$ to afford recovered cyclophane $\mathbf{1}(29 \mathrm{mg})$, octaherane 3 (19 $\left.\mathrm{mg}, 33 \%\right)$, and cage compound 4 (2 mg, 3\%). The yields were determined based on the consumed cyclophane 1 .

Physical data for octahedrane 3. Colorless prisms, mp 243-244 ${ }^{\circ} \mathrm{C},{ }^{1} \mathrm{H}$ NMR $\left(\mathrm{CDCl}_{3}, 600\right.$ MHz) $\delta 1.97\left(\mathrm{~m}, 4 \mathrm{H}, \mathrm{H}^{\mathrm{a}}\right), 3.35\left(\right.$ brs, $\left.2 \mathrm{H}, \mathrm{H}^{\mathrm{b}}\right), 3.68\left(\right.$ brs, $\left.2 \mathrm{H}, \mathrm{H}^{\mathrm{c}}\right), 3.74\left(\right.$ brs, $\left.2 \mathrm{H}, \mathrm{H}^{\mathrm{d}}\right) 3.75($ brd, $J$ $\left.=3.0 \mathrm{~Hz}, 2 \mathrm{H}, \mathrm{H}^{\mathrm{e}}\right), 3.77\left(\mathrm{brd}, J=2.3 \mathrm{~Hz}, 2 \mathrm{H}, \mathrm{H}^{\mathrm{f}}\right) .{ }^{13} \mathrm{C} \mathrm{NMR}\left(\mathrm{CDCl}_{3}, 150 \mathrm{MHz}\right) \delta 37.87\left(\mathrm{C}^{1 \mathrm{a}}\right)$, $38.03\left(\mathrm{C}^{1 \mathrm{~b}}\right), 47.9\left(\mathrm{C}^{2}\right), 48.03\left(\mathrm{C}^{3}\right), 51.9\left(\mathrm{C}^{4}\right), 53.2\left(\mathrm{C}^{5}\right), 53.5\left(\mathrm{C}^{6}\right), 54.0\left(\mathrm{C}^{7}\right), 70.2\left(\mathrm{C}^{8 \mathrm{a}}\right), 70.4$ $\left(\mathrm{C}^{8 \mathrm{~b}}\right), 76.5\left(\mathrm{C}^{9}\right), 79.3\left(\mathrm{C}^{10}\right), 116.3\left(\mathrm{q},{ }^{1} J_{\mathrm{CF}}=286 \mathrm{~Hz}, \mathrm{CF}_{3}\right), 116.2\left(\mathrm{q},{ }^{1} J_{\mathrm{CF}}=286 \mathrm{~Hz}, \mathrm{CF}_{3}\right)$, $155.24\left(\mathrm{q},{ }^{2} J_{\mathrm{CF}}=36 \mathrm{~Hz}, \mathrm{C}=\mathrm{O}\right), 155.21\left(\mathrm{q},{ }^{2} J_{\mathrm{CF}}=36 \mathrm{~Hz}, \mathrm{C}=\mathrm{O}\right) . \mathrm{IR}(\mathrm{KBr}) v_{\max } 1684,1456$, 1201, $1149 \mathrm{~cm}^{-1}$, Anal. Calcd. for $\mathrm{C}_{20} \mathrm{H}_{16} \mathrm{~F}_{6} \mathrm{~N}_{2} \mathrm{O}_{2}$ : C, 55.82; H, 3.75; N, 6.51. Found: C, 56.06; H, 3.68; N, 6.30.

Physical data for cage compound 4. Colorless crystals, mp $133-134{ }^{\circ} \mathrm{C},{ }^{1} \mathrm{H}$ NMR $\left(\mathrm{CDCl}_{3}, 600\right.$ MHz) $\delta 2.63\left(\mathrm{~m}, 4 \mathrm{H}, \mathrm{H}^{\mathrm{a}}\right) 3.28\left(\mathrm{~d}, J=12.6 \mathrm{~Hz}, 2 \mathrm{H}, \mathrm{H}^{\mathrm{b}}\right), 3.49\left(\mathrm{~d}, J=12.1 \mathrm{~Hz}, 2 \mathrm{H}, \mathrm{H}^{\mathrm{c}}\right), 4.01(\mathrm{~d}$, $\left.J=12.6 \mathrm{~Hz}, 2 \mathrm{H}, \mathrm{H}^{\mathrm{d}}\right), 4.32\left(\mathrm{~d}, J=12.6 \mathrm{~Hz}, 2 \mathrm{H}, \mathrm{H}^{\mathrm{e}}\right), 5.98\left(\mathrm{~s}, 2 \mathrm{H} / 2, \mathrm{H}^{\mathrm{f}}\right), 5.98(\mathrm{~d}, J=9.7 \mathrm{~Hz}$, $\left.2 \mathrm{H} / 2, \mathrm{H}^{\mathrm{f}}\right), 6.02\left(\mathrm{~s}, 2 \mathrm{H} / 2, \mathrm{H}^{\mathrm{g}}\right), 6.02\left(\mathrm{~d}, J=9.7 \mathrm{~Hz}, 2 \mathrm{H} / 2, \mathrm{H}^{\mathrm{g}}\right) .{ }^{13} \mathrm{C} \mathrm{NMR}\left(\mathrm{CDCl}_{3}, 150 \mathrm{MHz}\right) \delta$ $36.2\left(\mathrm{C}^{1 \mathrm{a}}\right), 36.6\left(\mathrm{C}^{1 \mathrm{~b}}\right), 55.6\left(\mathrm{C}^{2}\right), 57.2\left(\mathrm{C}^{3}\right), 57.4\left(\mathrm{C}^{4}\right), 57.6\left(\mathrm{C}^{5}\right), 116.2\left(\mathrm{CF}_{3},{ }^{1} J_{\mathrm{CF}}=278 \mathrm{~Hz}\right)$, $131.6\left(C^{7 a}\right), 132.1\left(C^{7 b}\right), 155.5(C=O) . I R(K B r) v_{\max } 1694,1464,1211,1183,1149,1135 \mathrm{~cm}^{-1}$. Anal. Calcd. for $\mathrm{C}_{20} \mathrm{H}_{16} \mathrm{~F}_{6} \mathrm{~N}_{2} \mathrm{O}_{2}$ : C, 55.82; H, 3.75; N, 6.51. Found: C, 55.71; H, 3.66; N, 6.07. For the assignments of the ${ }^{1} \mathrm{H}$ and ${ }^{13} \mathrm{C}$ signals, see Chart $\mathrm{S} 1$. 
CHART S1. Assignment of the ${ }^{1} \mathrm{H}$ and ${ }^{13} \mathrm{C}$ NMR spectra of octahedrane 3 and cage compound 4 .

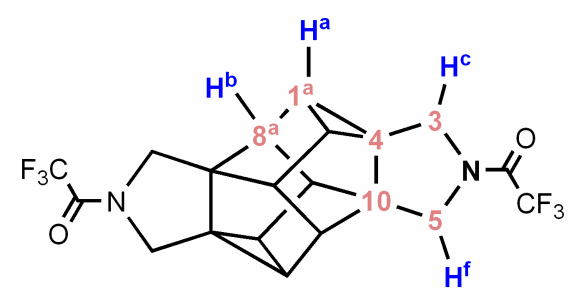

3 (conformer a)

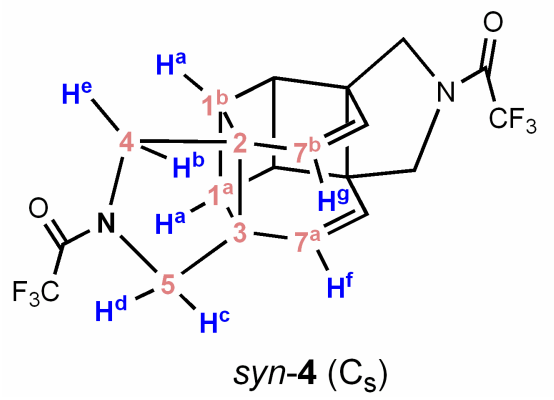

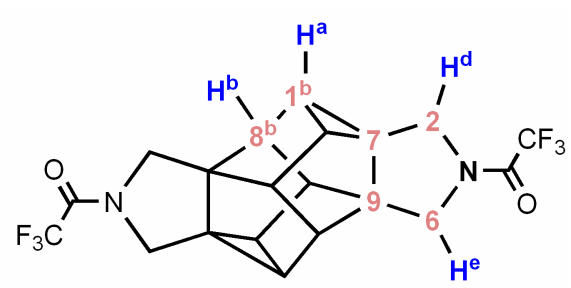

3 (conformer b)

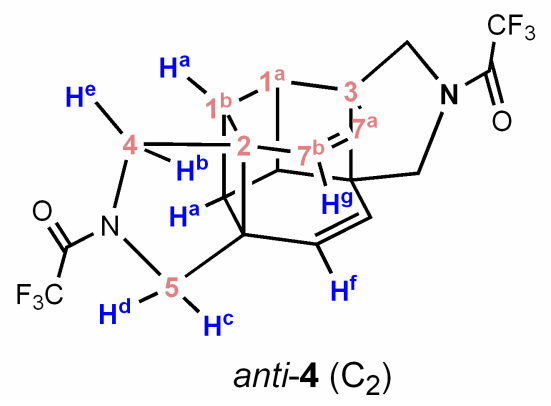




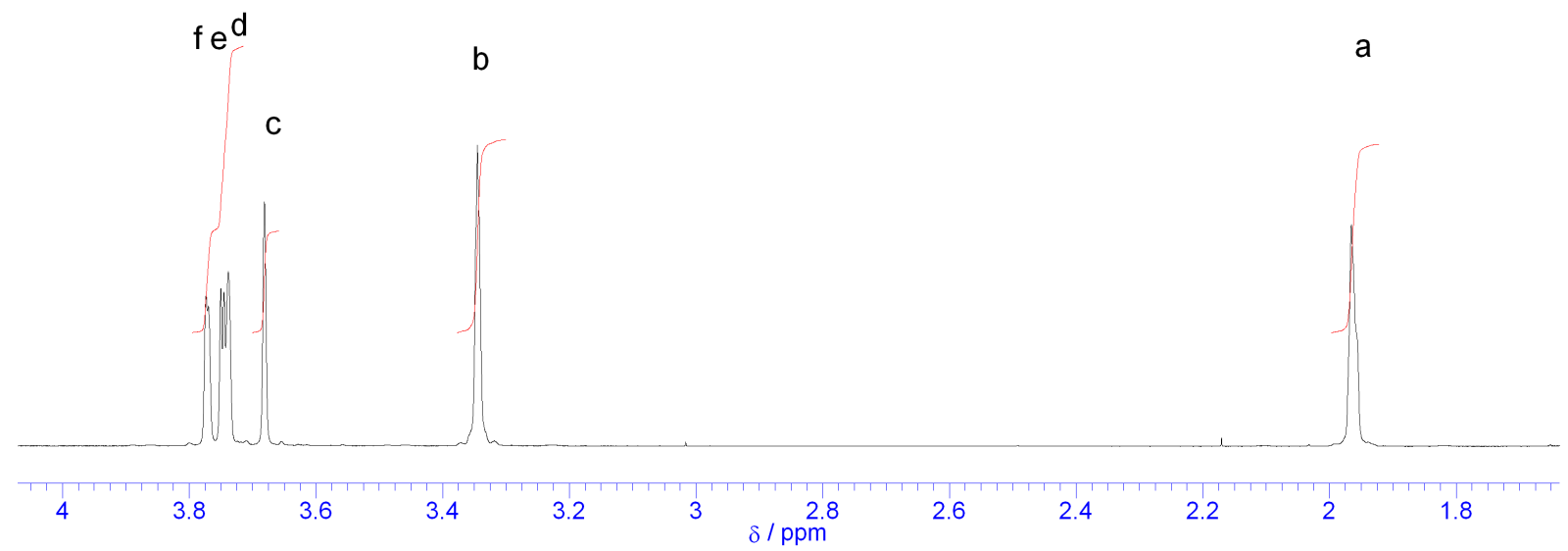

FIGURE S1. ${ }^{1} \mathrm{H}$ NMR spectrum of octahedrane $3\left(600 \mathrm{MHz}, \mathrm{CDCl}_{3}\right)$. For the assignments of the signals, see CHART S1. 


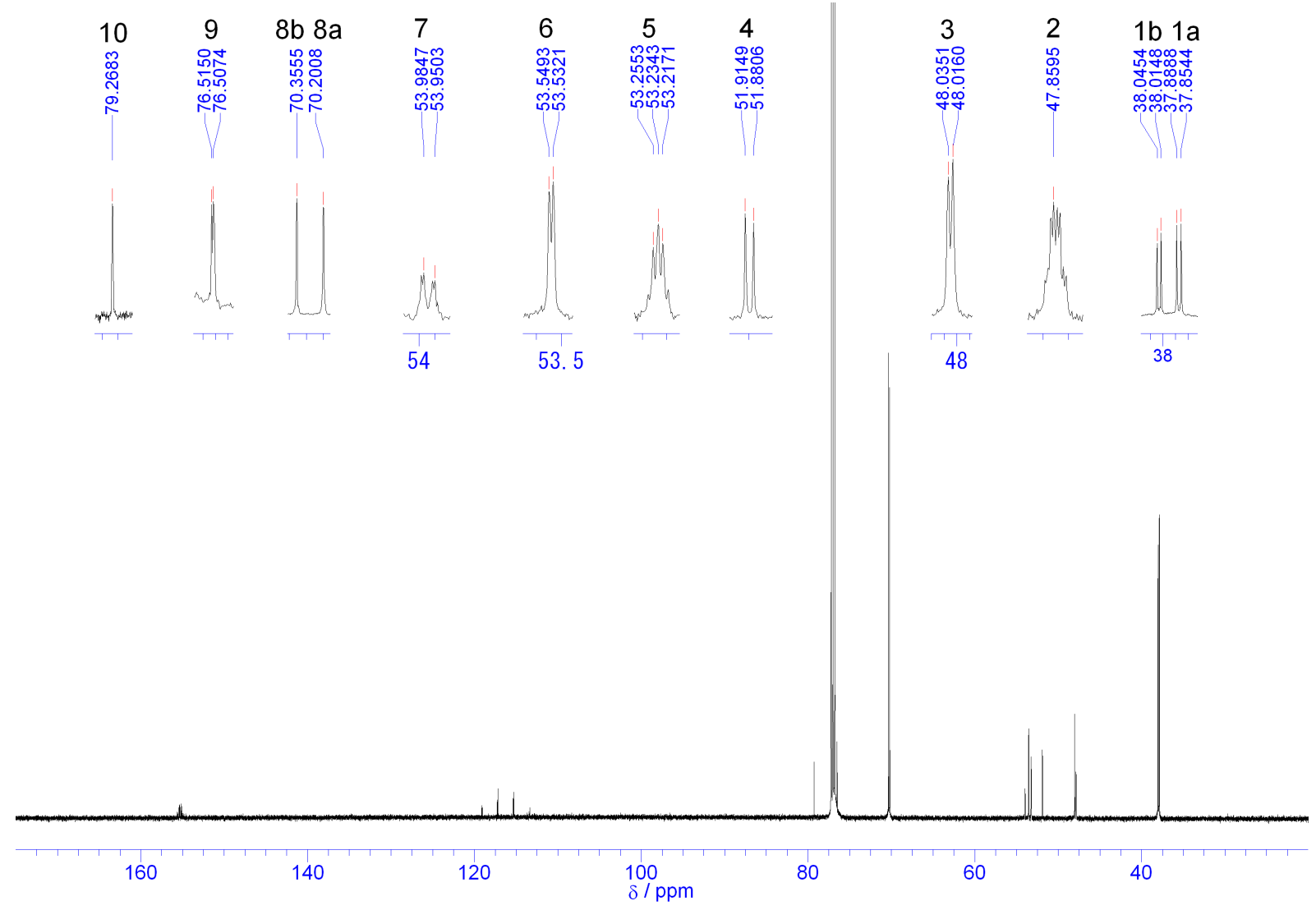

FIGURE S2. ${ }^{13} \mathrm{C}$ NMR spectrum of octahedrane $3\left(150 \mathrm{MHz}, \mathrm{CDCl}_{3}\right)$. For the assignments of the signals, see CHART S1. 


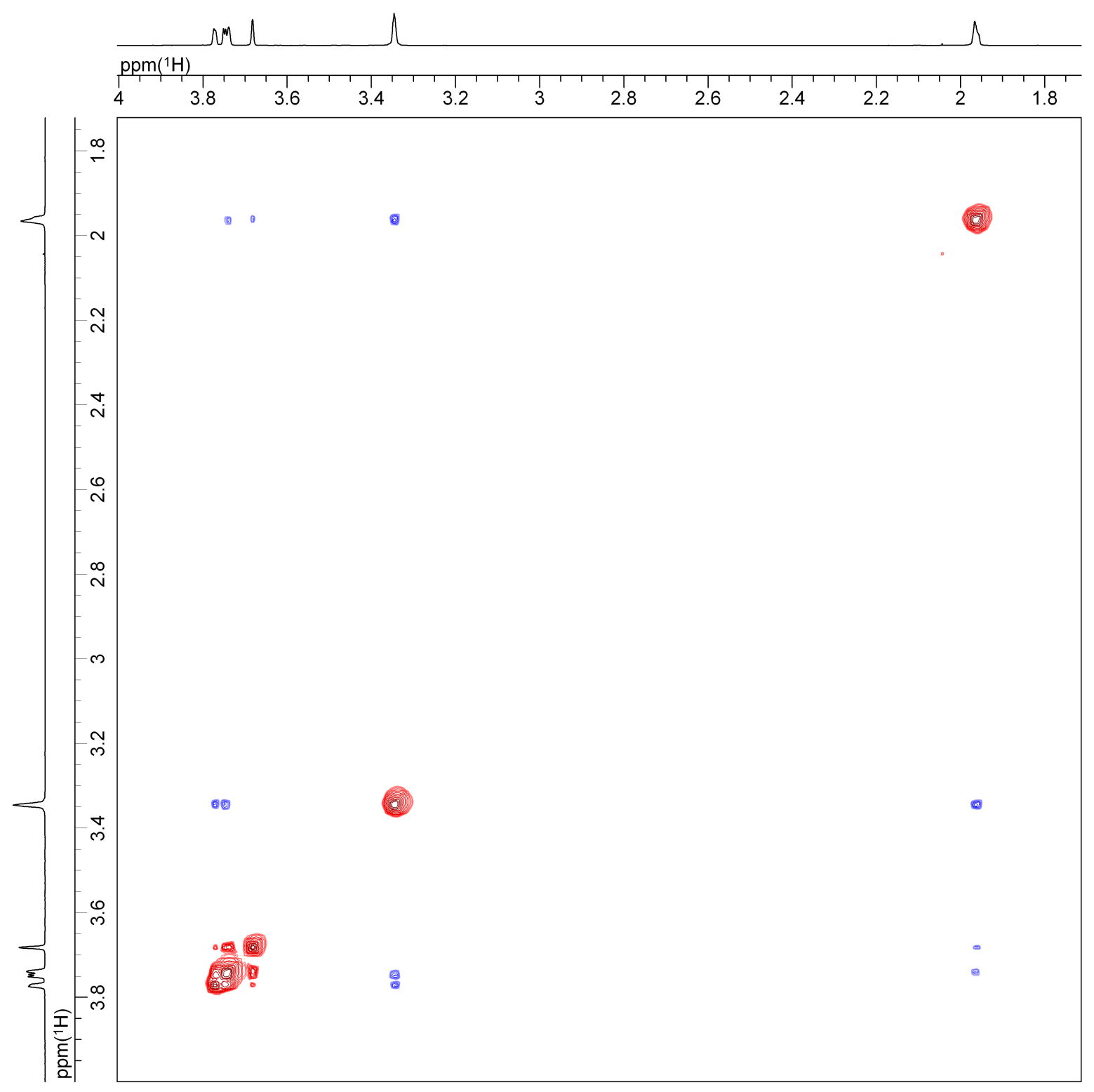

FIGURE S3. NOESY spectrum of octahedrane $3\left(600 \mathrm{MHz}, \mathrm{CDCl}_{3}\right)$. The blue and red cross peaks indicate positive (NOE) and negative (exchange) signals, respectively. 


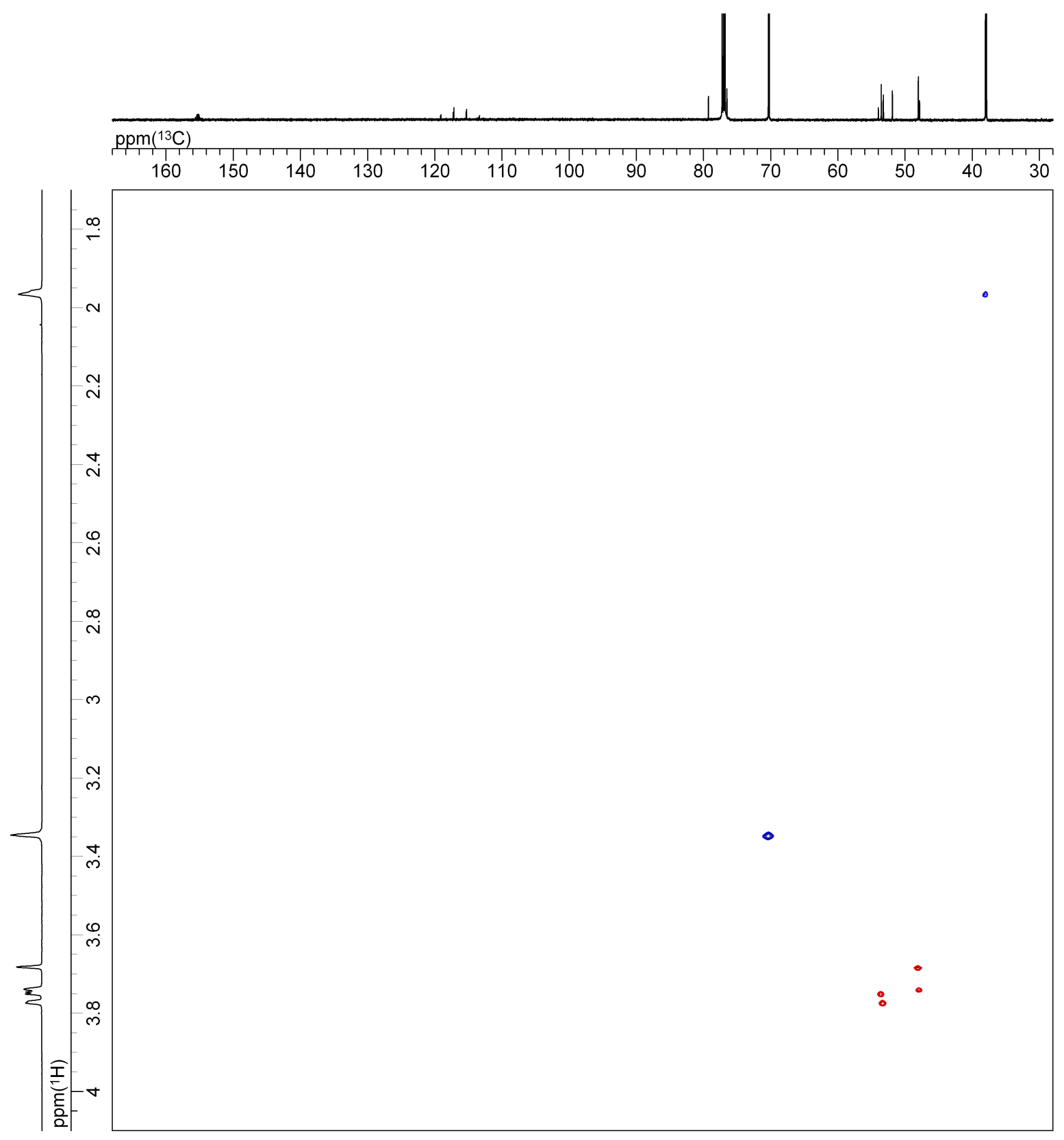

FIGURE S4. HSQC spectrum of octahedrane $3\left(600 \mathrm{MHz}, \mathrm{CDCl}_{3}\right)$. The blue and red cross peaks indicate positive (methine) and negative (methylene) signals, respectively. 


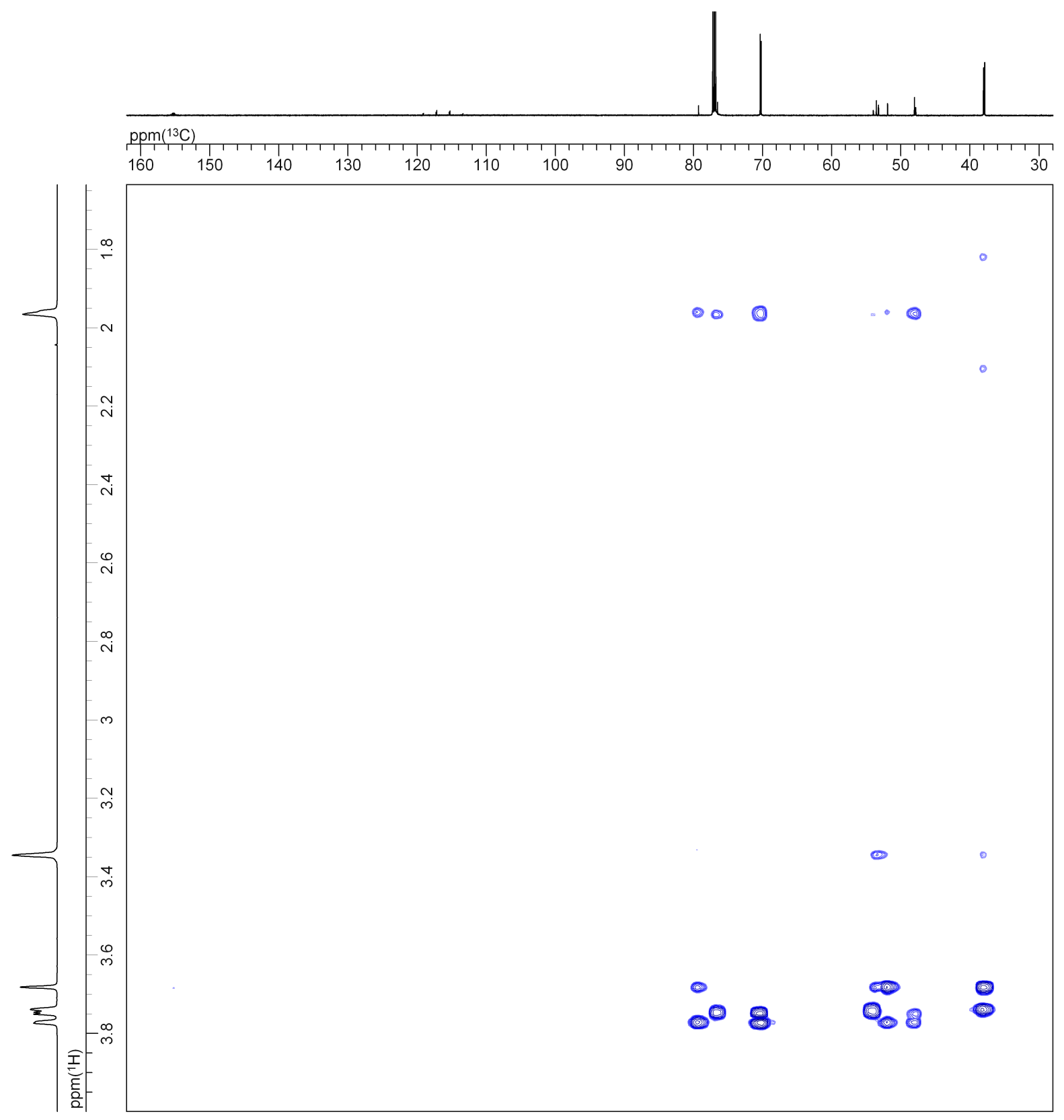

FIGURE S5. HMBC spectrum of octahedrane $3\left(600 \mathrm{MHz}, \mathrm{CDCl}_{3}\right)$. 


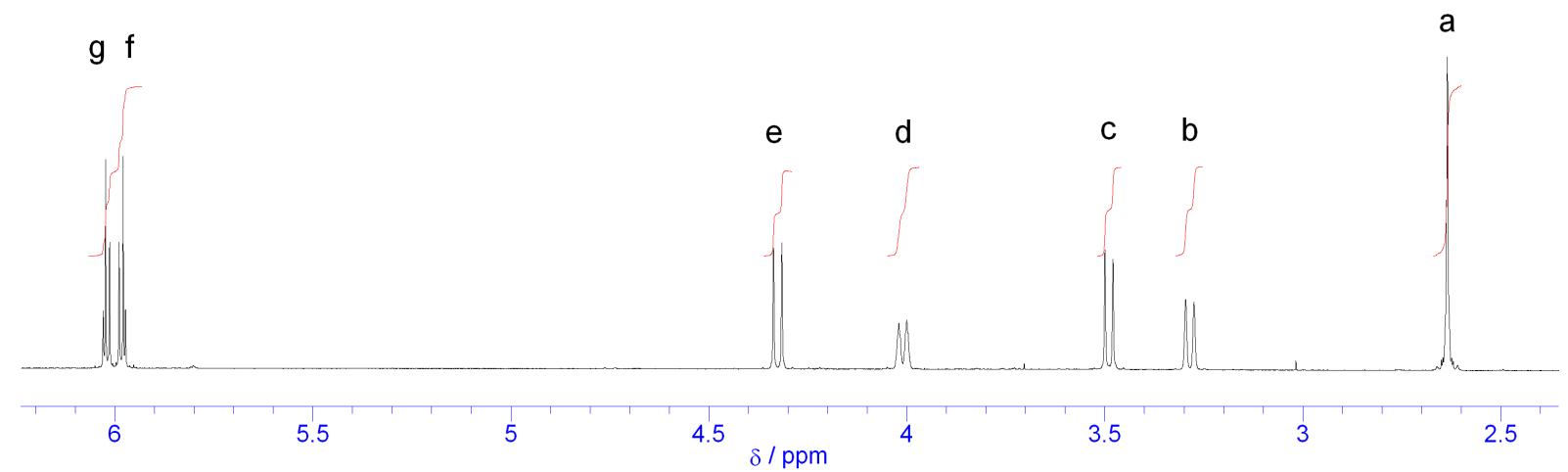

FIGURE S6. ${ }^{1} \mathrm{H}$ NMR spectrum of cage compound $4\left(600 \mathrm{MHz}, \mathrm{CDCl}_{3}\right)$. For the assignments of the signals, see CHART S1. 


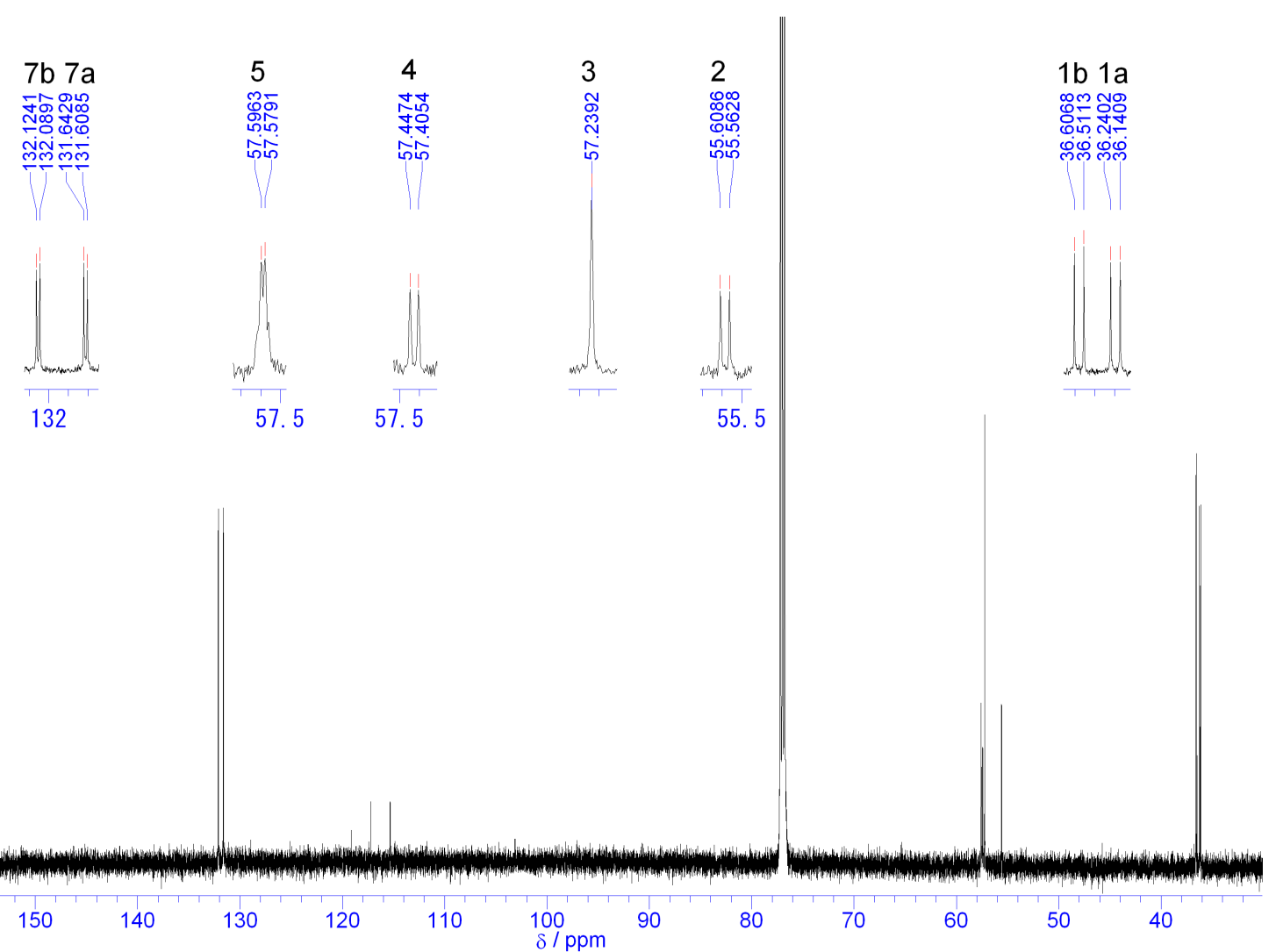

FIGURE S7. ${ }^{13} \mathrm{C}$ NMR spectrum of cage compound $4\left(150 \mathrm{MHz}, \mathrm{CDCl}_{3}\right)$. For the assignments of the signals, see CHART S1. 


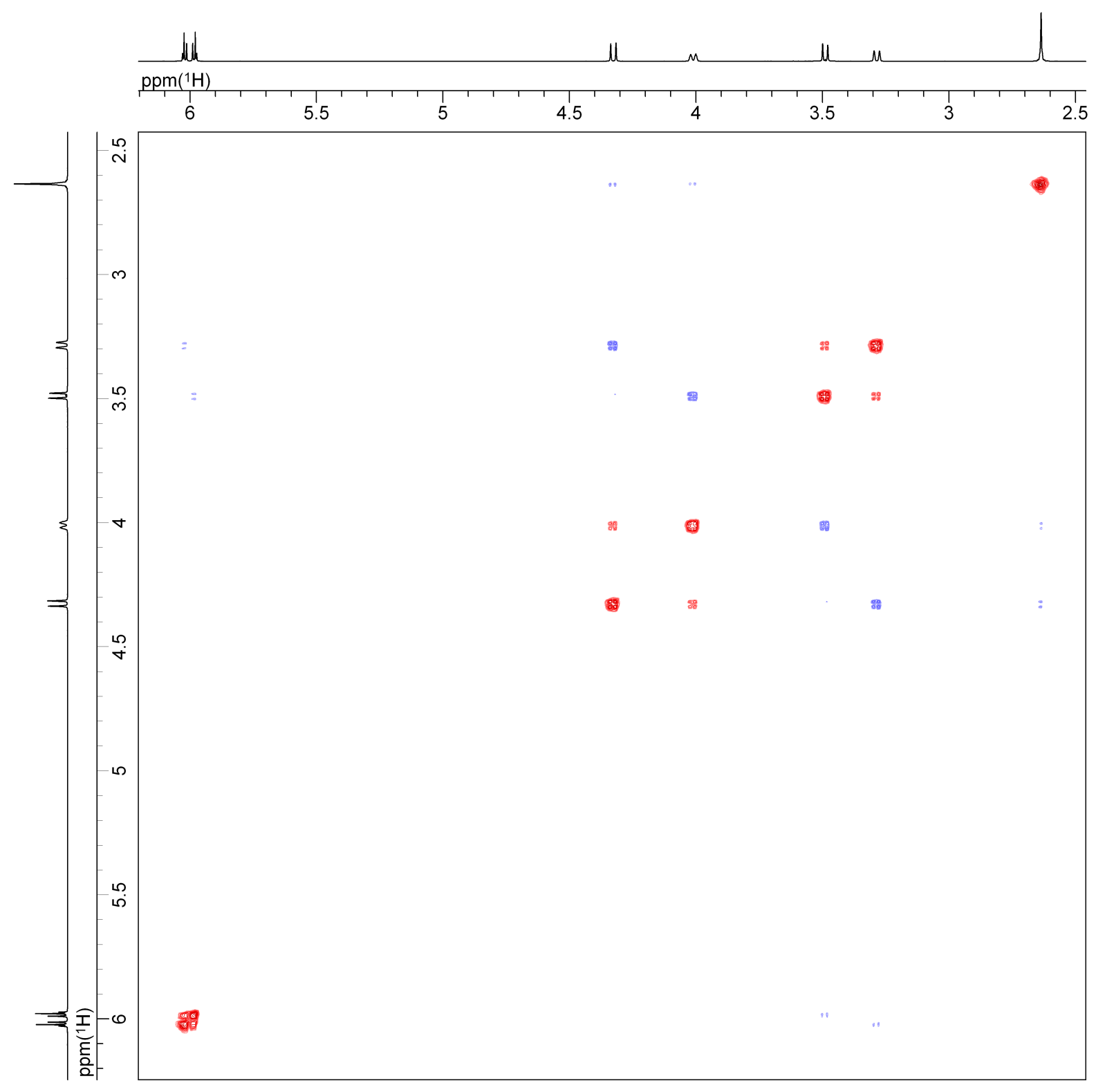

FIGURE S8. NOESY spectrum of cage compound $4\left(600 \mathrm{MHz}, \mathrm{CDCl}_{3}\right)$. The blue and red cross peaks indicate positive (NOE) and negative (exchange) signals, respectively. 


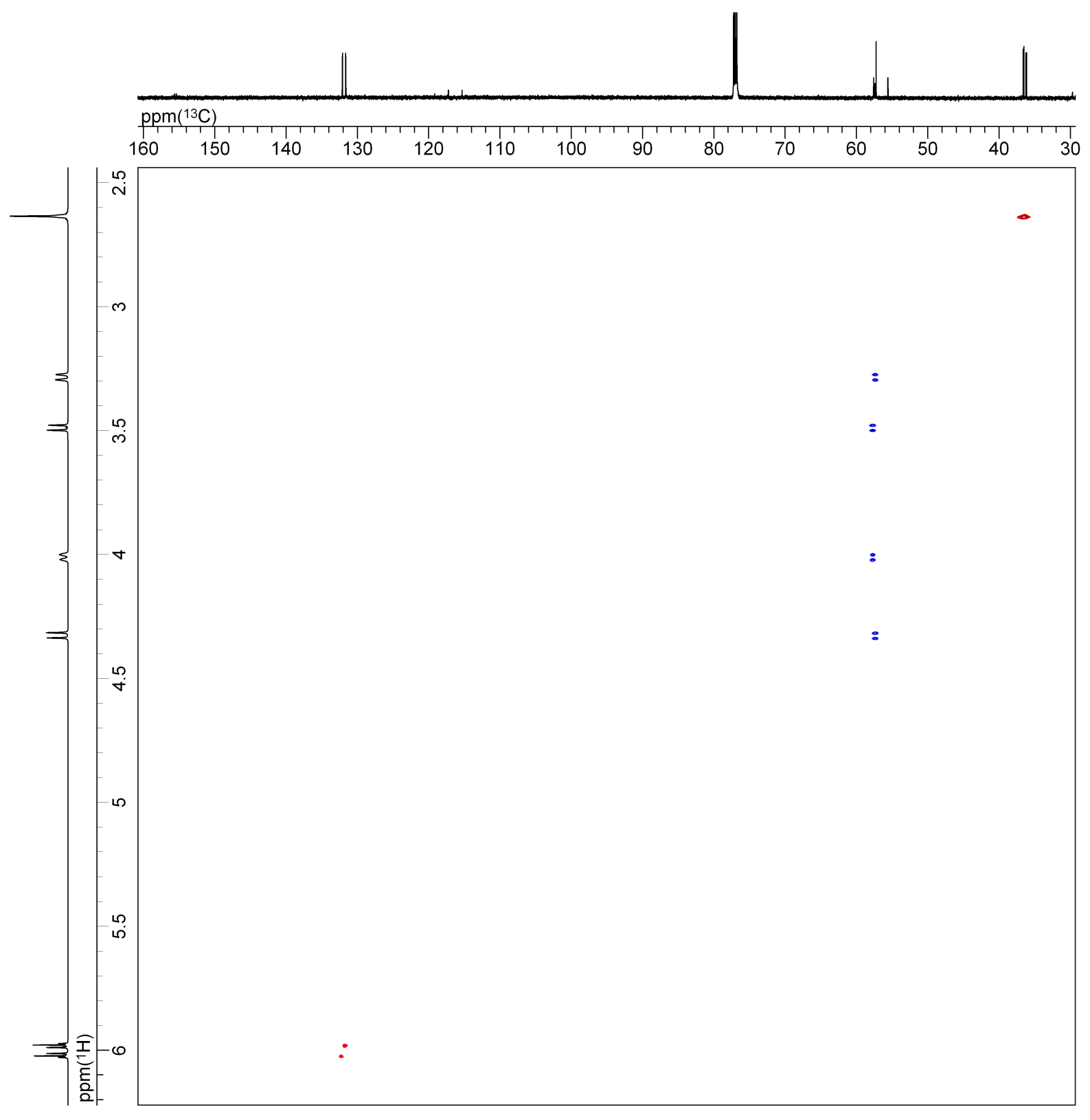

FIGURE S9. HSQC spectrum of cage compound $4\left(600 \mathrm{MHz}, \mathrm{CDCl}_{3}\right)$. The blue and red signals indicate positive (methine) and negative (methylene) cross peaks, respectively. 


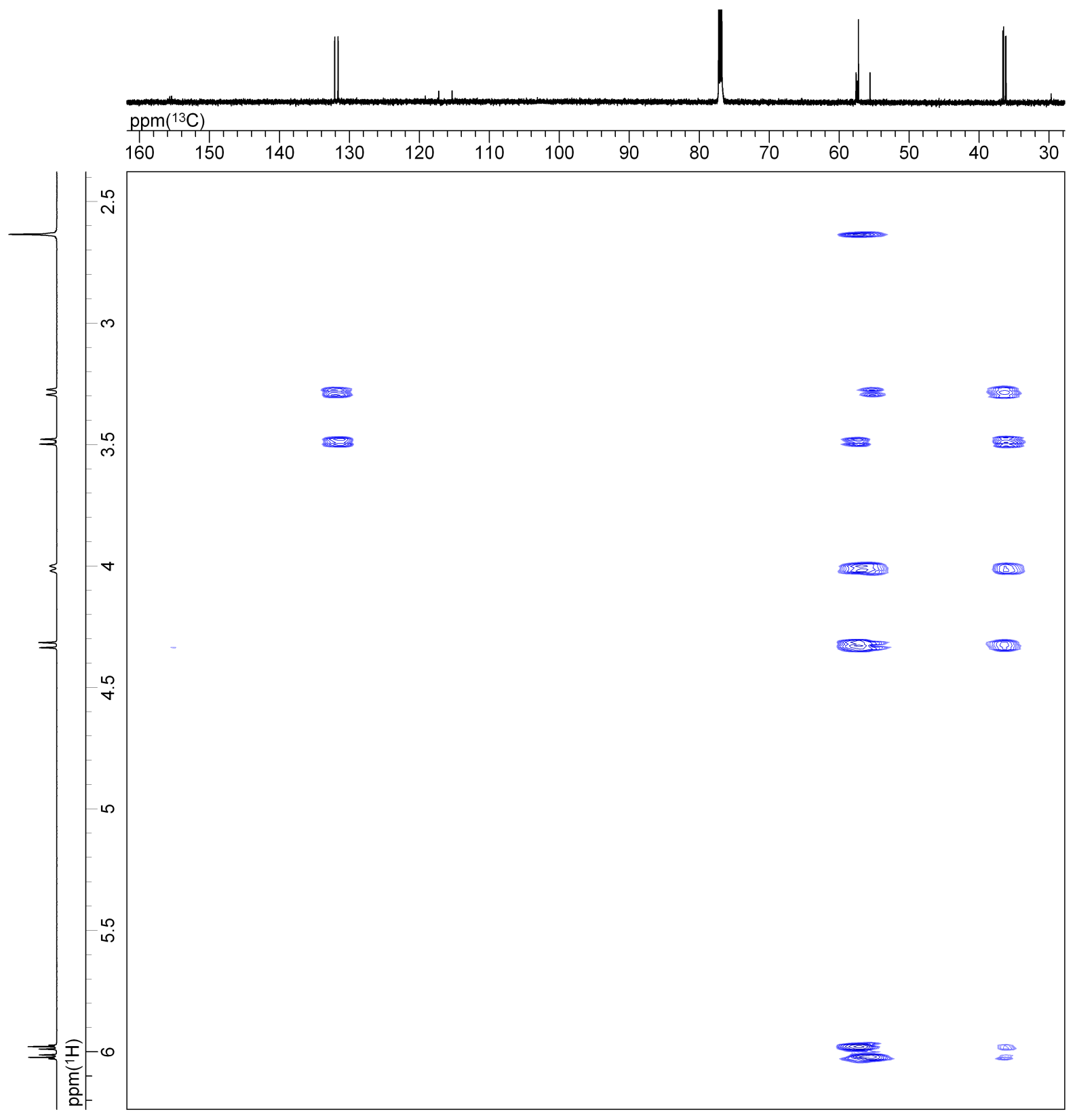

FIGURE S10. HMBC spectrum of cage compound $4\left(600 \mathrm{MHz}, \mathrm{CDCl}_{3}\right)$. 
X-ray crystallographic study of octahedrane 3.

The X-ray structural analysis of octahedrane $\mathbf{3}$ was performed at $173 \mathrm{~K}$ using a Rigaku RAXIS-RAPID II diffractometer (graphite-monochromated Mo-K $\mathrm{K}_{\alpha}, \lambda=0.71075 \AA$ ) equipped with an imaging plate area detector. The crystal structure was solved by direct method, SHELXS $97^{3}$ and refined by the full-matrix least-squares method, SHELXL97. ${ }^{3}$

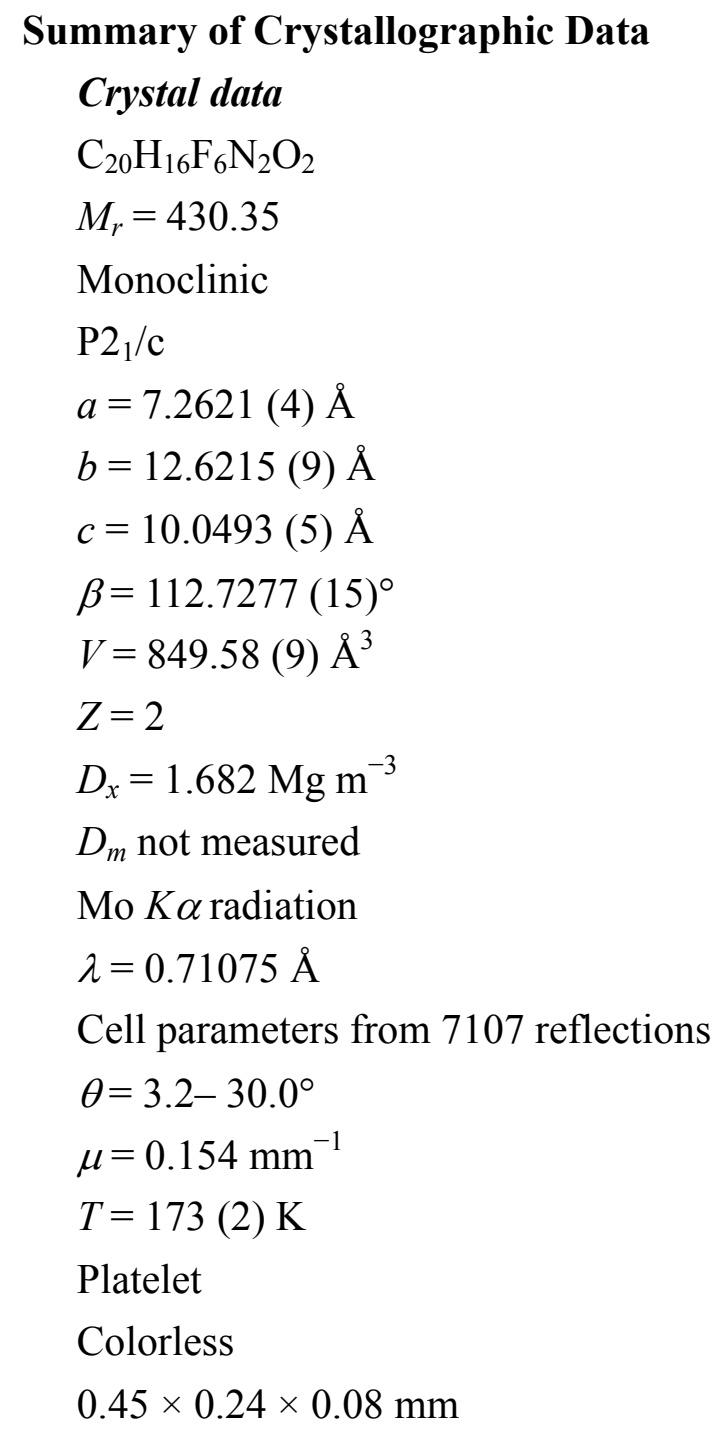

\section{Data collection}

Rigaku RAXIS-RAPID II diffractometer $\omega$ scans

Absorption correction:

multi-scan (ABSCOR; Higashi, 1995)

$$
T_{\min }=0.782, T_{\max }=0.988
$$


315 measured reflections

2460 independent reflections

1877 reflections with $>2 \sigma(I)$

$R_{\text {int }}=0.0376$

$\theta_{\max }=29.98^{\circ}$

$h=-8 \rightarrow 10$

$k=-17 \rightarrow 17$

$l=-14 \rightarrow 13$

\section{Refinement}

Refinement on $F^{2}$

$R(F)=0.0474$

$w R\left(F^{2}\right)=0.1293$

$S=1.106$

2460 reflections

169 parameters

All H-atom parameters refined

$w=1 /\left[\sigma^{2}\left(F_{o}^{2}\right)+(0.0652 P)^{2}+0.1356 P\right]$

where $P=\left(F_{o}^{2}+2 F_{c}^{2}\right) / 3$

$(\Delta / \sigma)_{\max }<0.001$

$\Delta \rho_{\max }=0.295$ e $\AA^{-3}$

$\Delta \rho_{\text {min }}=-0.344$ e $\AA^{-3}$

Extinction correction: none

Scattering factors from International Tables for Crystallography (Vol. C) 


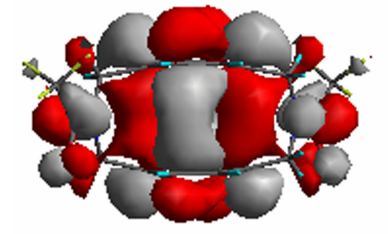

(a)

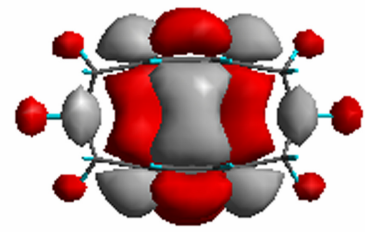

(b)

FIGURE S12. LUMO figures of cyclophanes (a) 1 and (b) 2 calculated by DFT method at B3LYP/6-31+G(d) level of theory. 


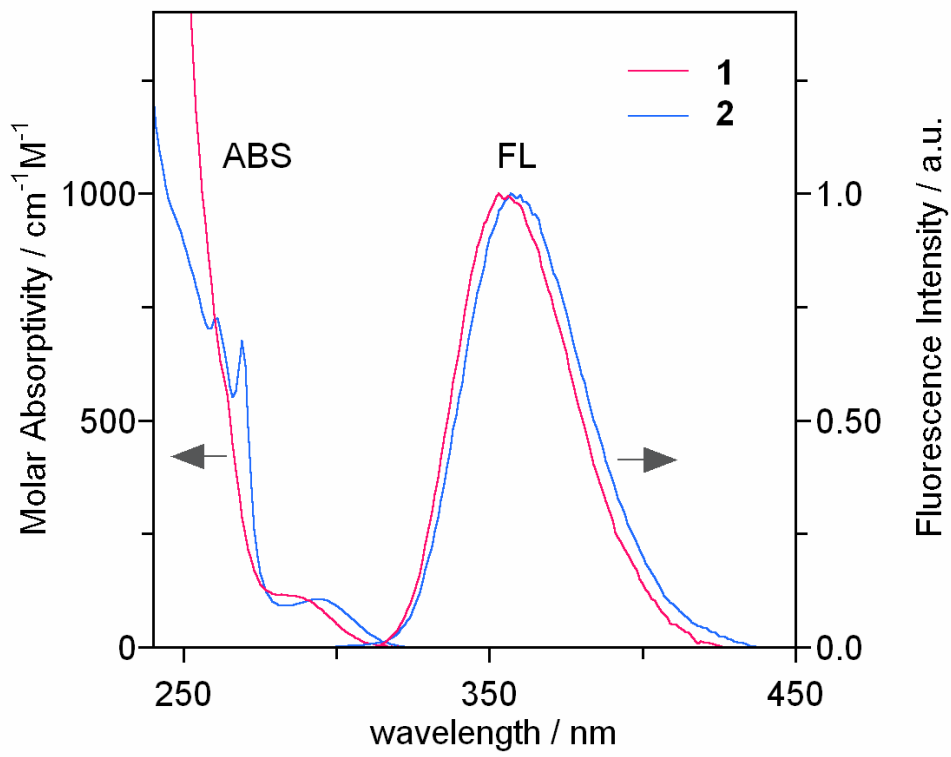

FIGURE S13. Absorption and fluorescence (normalized, $\lambda_{\mathrm{ex}} 265 \mathrm{~nm}$ ) spectra of cyclophanes 1 and 2 in MeCN. Fluorescence quantum yield of $\mathbf{1}$ and 2 is $3 \times 10^{-3}$ and $8 \times 10^{-3}$ (errors $\pm 10 \%$ ) respectively. 


\section{Additional References.}

(1) Pereira, L. C.; Ferreira, I. C.; Thomaz, M. P. F. Chem. Phys. Lett. 1976, 43, 157-161.

(2) Gaussian 03, Revision B.03, Frisch, M. J.; Trucks, G. W.; Schlegel, H. B.; Scuseria, G. E.; Robb, M. A.: Cheeseman, J. R.; Montgomery, Jr., J. A.; Vreven, T.; Kudin, K. N.; Burant, J. C.; Millam, J. M.; Iyengar, S. S.; Tomasi, J.; Barone, V.; Mennucci, B.; Cossi, M.; Scalmani, G.; Rega, N.; Petersson, G. A.; Nakatsuji, H.; Hada, M.; Ehara, M.; Toyota, K.; Fukuda, R.; Hasegawa, J.; Ishida, M.; Nakajima, T.; Honda, Y.; Kitao, O.; Nakai, H.; Klene, M.; Li, X.; Knox, J. E.; Hratchian, H. P.; Cross, J. B.; Adamo, C.; Jaramillo, J.; Gomperts, R.; Stratmann, R. E.; Yazyev, O.; Austin, A. J.; Cammi, R.; Pomelli, C.; Ochterski, J. W.; Ayala, P. Y.; Morokuma, K.; Voth, G. A.; Salvador, P.; Dannenberg, J. J.; Zakrzewski, V. G.; Dapprich, S.; Daniels, A. D.; Strain, M. C.; Farkas, O.; Malick, D. K.; Rabuck, A. D.; Raghavachari, K.; Foresman, J. B.; Ortiz, J. V.; Cui, Q.; Baboul, A. G.; Clifford, S.; Cioslowski, J.; Stefanov, B. B.; Liu, G.; Liashenko, A.; Piskorz, P.;. Komaromi, I; Martin, R. L.; Fox, D. J.; Keith, T.; Al-Laham, M. A.; Peng, C. Y.; Nanayakkara, A.; Challacombe, M.; Gill, P. M. W.; Johnson, B.; Chen, W.; Wong, M. W.; Gonzalez, C.; Pople, J. A. Gaussian, Inc., Pittsburgh PA, 2003.

(3) Sheldrick, G. M. Programs for the Solution of Crystal Structures; University of Göttingen, Germany, 1997. 